\title{
La percepción de la conflictividad en el profesorado de Educación Secundaria: estudio de caso
}

\section{The perception of conflictivity in the Secondary Education teacher: case study}

\author{
MARÍA ISABEL ROJO GUILLAMÓN \\ Experta en mediación, doctoranda en educación, directora y orientadora del Colegio Concertado EL TALLER de Moli- \\ na de Segura (Murcia).mrg64433@um.es.https://orcid.org/0000-0002-2030-9537
}

\begin{abstract}
Resumen: El objetivo de este trabajo es conocer y analizar los tipos de conflictos entre el profesorado de un Instituto de Enseñanza Secundaria (en adelante IES) en la Región de Murcia. Los instrumentos utilizados han seguido una doble metodología. Por una parte, una metodología cuantitativa con aplicación de la técnica de la encuesta y por otra parte, con una metodología cualitativa en aplicación de las técnicas de grupo de discusión y entrevistas semiestructuradas. Los participantes fueron 35 docentes seleccionados sobre una población total de 92. Los resultados indican que los tipos de conflictos principales son: discrepancias y desacuerdos entre estos y el equipo directivo y la administración. Al mismo tiempo, otro de los tipos de conflictos que se generan es la distribución de horarios y las franjas horarias en las que el profesorado desea impartir las asignaturas. Igualmente, encontramos que el profesorado percibe el conflicto de manera distinta en función de la edad, así pues, el profesorado con edades comprendidas entre los 36 y 45 años prefiere evitar el conflicto, frente al grupo del profesorado de mayor edad que percibe el conflicto como "lucha para vencer". Sin embargo, el grupo de profesorado más joven y el grupo con mayor experiencia docente, lo percibe como oportunidad de cambio, desde el punto de vista positivo. El sexo femenino ante el conflicto, prefiere bien, ceder o ignorar a la otra parte. Siendo la principales causas de los conflictos: ideológicas, comunicación deficiente, insatisfacción laboral o diferencia de funciones.
\end{abstract}

Palabras clave: Enseñanza Secundaria, conflictos, profesorado, insatisfacción laboral.

\begin{abstract}
The objective of this research is to identify and analyze the types of conflicts between the teaching staff of a Secondary Education School (referred to hereafter as IES) in the Region of Murcia. We have used a double methodology. On the one hand, a quantitative methodology with the application of a survey. On the other hand, the application of a qualitative methodology using the techniques of group discussion and semi-structured interviews. The participants were 35 selected teachers out of a total of 92 teachers. The results indicate that the main types of conflicts were: discrepancies and disagreements between the teaching staff and the management team and regional administration. At the same time, other types of conflicts are generated by the distribution of timetables and the time slots in which the teaching staff would want to teach their subjects. Likewise, we found that teachers
\end{abstract}


perceive conflict differently according to their age. Therefore, teachers aged between 36 and 45 prefer to avoid conflict, compared to the group of older teachers who perceive the conflict as "a fight to win". However, the group of younger teachers and the group of the most experienced teachers both perceive conflict as an opportunity for change, from a positive point of view. When female teachers face conflict, they prefer either to yield or ignore the other side of the conflict. The main causes conflict are usually ideological, poor communication, job dissatisfaction or differences in roles and responsibilities at work.

Key words: Secondary education, conflicts, teachers, job dissatisfaction.

\section{PLANTEAMIENTO DEL PROBLEMA/INTRODUCCIÓN}

Los conflictos entre el profesorado de los IES se enmarcan en un contexto educativo, por lo tanto, cabe entender que éstos se sitúan dentro de lo que tradicionalmente se ha ido denominando conflictos escolares. Si bien es cierto que, las relaciones laborales entre los trabajadores producen en algunos casos conflictos y riesgos psicosociales con consecuencias negativas en la organización y productividad (Camacho y Mayorga, 2017), no se considerará en este estudio por tratarse de conflictos laborales y no escolares.

Aunque los estudios sobre conflictos escolares están fundamentalmente centrados en las malas relaciones entre alumnado, los conflictos entre los docentes forman parte de la vida de los IES. Puesto que, la misma naturaleza convivencial de los miembros de la comunidad educativa hace que las relaciones puedan debilitarse, romperse o perturbarse de forma considerable, dando lugar a conflictos de cualquier índole, por lo que debe formar parte de los propósitos educativos (Martínez-Otero, 2001). Resulta contradictorio que en un contexto educativo el profesorado, sintiéndose más especialista que educador, no sea capaz en algunos casos de gestionar los conflictos entre compañeros, en perjuicio del clima educativo. Si los educadores entendieran el conflicto como algo positivo, sería posible trabajar con él y desde él, aprovechando la oportunidad educativa que brinda (Pérez y Pérez de Guzmán, 2011). Los conflictos entre docentes no solo afectan al desempeño de su trabajo y al nivel de satisfacción, sino también a aspectos organizativos, relacionales y curriculares, sin olvidar la incidencia en el alumnado y su rendimiento académico (Benito, 2006).

Entre los componentes del conflicto se destaca por una parte, las dimensiones relativas al proceso cognitivo-conductual y a las relaciones de poder que se corresponden con la satisfacción de las necesidades en los procesos de estrés, sensaciones de temor y en el desarrollo de la acción que pueden ocasionar comportamientos agresivos y violentos (Redorta, 2006; Vinyamata, 2003).Y por otra parte, el conflicto es abordado desde su funcionalidad, en la búsqueda de resolu- ción de metas opuestas con valores antagónicos o intereses divergentes (Jares 2001; Merlano, 2011). Considerando el conflicto como contraposición a alguien o algo y como proceso que modifica actitudes, emociones y decisiones.

Los conflictos en la comunidad educativa son consustanciales a la misma, por tanto también lo son entre el profesorado y más concretamente en los IES de la Región de Murcia; sin embargo, su tratamiento documental no siempre significativo, ha sido escaso. Los datos reflejados en la memoria del sindicato "Asociación Nacional de Profesionales de la Enseñanza" (2011-12), ponen de manifiesto que en los centros educativos se producen conflictos entre docentes en todos los niveles educativos, incluida la educación universitaria. Algunos de los factores que influyen y propician situaciones de enfrentamiento entre el profesorado son: los problemas para compartir espacios comunes, intereses a veces contrapuestos y los daños colaterales producidos por los distintos recortes aplicados en educación; así mismo, la elección de horarios y tutorías, la falta de personal, funciones distintas e intereses enfrentados, generan malestar y animadversión. Situaciones que dejan de ser puntuales para convertirse en sistemáticas, lo que propicia estrés para el docente que las sufre.

El profesorado (sigue recogiendo la memoria) se queja de que los equipos directivos hacen caso omiso de la normativa general que regula la asignación de tutorías, horarios y elección de grupos, en perjuicio de aquellos docentes que por no entrar en conflicto, optan por el aislamiento. Igualmente, perciben que en situaciones de conflicto no encuentran respaldo por los equipos directivos, eludiendo de esta manera los problemas que pudieran surgir. Además, se recoge que algunas causas son la falta de vocación hacia la enseñanza, rasgos de personalidad distintos, falta de compromisos, individualismo, diversidad de metas y falta de coordinación, que deriva en insatisfacción docente, estrés y agotamiento profesional producido por tensiones; con poca preparación en el uso de técnicas para afrontar las situaciones conflictivas.

Para Tahull y Montero (2015), esta realidad de los centros educativos ha sido escasamente abordada y ante un aparente 
clima positivo, aparecen interacciones entre el profesorado y equipo directivo insatisfactorias que dan lugar a estrés, depresión, ansiedad o acoso que producen un bajo nivel de la enseñanza. Unidos a otros factores y dificultades tales, como las conductas disruptivas de los alumnos y la falta de implicación familiar, generando insatisfacción, malestar docente y desgaste profesional (Hermosa, 2006; Arias y Jiménez, 2013; Torres, 2013; Sánchez, March y Ballester, 2015; González, 2017).

En este sentido, el profesorado de Educación Secundaria Obligatoria (en adelante ESO) se convierte en población de riesgo para desarrollar algunos tipos de trastornos de salud mental, sobre todo en aquellos docentes que no se sienten respaldados por el equipo directivo, cuando se ven envueltos en conflicto (Galdeano, Godoy y Cruz, 2007). Igualmente, se produce insatisfacción del profesorado cuando no obtiene soluciones desde la administración educativa (Muñoz-Méndez et al. 2017).

A estas situaciones que provocan conflictividad entre el profesorado, se añade la cantidad de alumnos por grupo (imposibilidad de lo que supone atender sus necesidades), falta de vocación docente, impartir docencia distinta a la especialidad que se ha estudiado y la sobrecarga escolar (reuniones y diversidad de tareas administrativas) que originan bajas psiquiátricas alterando la organización del centro educativo y las programaciones docentes, con efectos negativos también para el alumnado (Prieto y Bermejo, 2006).

Por ello, el interés de este trabajo obedece a la repercusión de estos conflictos escolares en la labor y responsabilidad docente que desempeñan. El estudio se realizó en el IES Francisco de Goya, centro de titularidad pública situado en una zona residencial de Molina de Segura (Murcia), con una población de unos 71.000 habitantes. ${ }^{1}$ Con 910 alumnos de un nivel sociocultural medio, de los que 160 son de nacionalidad extranjera, representado un 17,5\% de la totalidad. La relación de cargos unipersonales del profesorado la compone el equipo directivo. Con 92 docentes que forman la plantiIla. ${ }^{2}$ Fue elegido por ser uno de los centros con mayor oferta educativa del municipio (ciclos formativos de grado medio y superior, formación profesional básica, ESO y bachiller).

\section{OBJETIVO}

El objetivo del estudio es doble: se trata de analizar la percepción de la conflictividad en el profesorado y averiguar los tipos de conflictos más frecuentes que se generan entre los docentes del IES Francisco de Goya de Molina de Segura (Murcia) derivados de su labor escolar y profesional.

\section{METODOLOGÍA}

Para Tamayo (2002:37), "la investigación es un proceso que, mediante la aplicación del método científico, procura obtener información relevante y fidedigna, para entender, verificar, corregir o aplicar el conocimiento". Todo ello requiere, de un planteamiento y la definición del método científico que vamos a utilizar. De tal manera que, se ha utilizado una metodología mixta con aplicación de: análisis documental, grupo de discusión, encuesta y entrevistas semiestructuradas.

La muestra elegida para llevar a cabo el grupo de discusión, fueron 7 jefes de departamento (matemáticas, historia, inglés, lengua, informática, tecnología y cultura clásica). Se consideró que estos no debían participar en el cuestionario posterior, para considerar la opinión del profesorado y no la opinión de los miembros del staff.

La muestra elegida para la encuesta fueron 35 docentes (62,9 \% hombres y 37, $1 \%$ mujeres), pertenecientes a distintos departamentos didácticos (matemáticas, lengua, informática, latín, cultura clásica, física y química). La selección se realizó con el listado de un universo de 92. Se eligió uno de cada tres docentes de forma probabilística, para que todos los elementos de la población tuvieran la misma probabilidad de ser escogidos, utilizando el azar estadístico. En este sentido, el profesorado impartía docencia en ciclos formativos, ESO y bachiller (la mayor parte de la muestra), mostrado en la Tabla I.

\begin{tabular}{|lcc|}
\hline \multicolumn{3}{|c|}{ TABLA I. Docencia IMPARTIDA POR LOS PROFESORES PARTICIPANTES } \\
\hline Ciclos formativos & Frecuencia & Porcentaje \\
\hline ESO & 5 & 14,3 \\
\hline Bachiller & 4 & 11,4 \\
\hline Total & 26 & 74,3 \\
\hline
\end{tabular}

Se formaron tres grupos en función de la edad (entre los 25 y 60 años), mostrado en la Tabla II.

\begin{tabular}{lcc}
\multicolumn{3}{c}{ Tabla II. GRUPOS En fUnCión De LA EDAD DE LOS PARTICIPANTES } \\
& Frecuencia & Porcentaje \\
\hline de 25 a 35 años & 2 & 5,7 \\
\hline de 36 a 45 & 12 & 34,3 \\
\hline de 46 a 60 & 21 & 60,0 \\
\hline Total & 35 & 100,0
\end{tabular}

1 Fuente: para el desarrollo de este apartado se ha consultado la Página Web del Instituto Francisco de Goya de Molina de Segura.

2 Fuente: para el desarrollo de este apartado se ha consultado la Programación General Anual (PGA), el Proyecto Educativo de Centro (PEC). 
La muestra elegida para las entrevistas semiestructuradas fueron los miembros de cada departamento didáctico (matemáticas, historia, latín, química, física, filosofía, lengua, francés, inglés y economía). El perfil de los participantes se muestra en la Tabla III.

\begin{tabular}{|c|c|c|}
\hline \multicolumn{3}{|c|}{$\begin{array}{l}\text { TABLA III. PERFIL DE LOS PARTICIPANTES EN } \\
\text { LAS ENTREVISTAS SEMIESTRUCTURADAS }\end{array}$} \\
\hline Departamento & Código & Perfil de los participantes \\
\hline Matemáticas & E1 & $\begin{array}{l}\text { Profesor de matemáticas, con } \\
35 \text { años de edad y siete años } \\
\text { de experiencia. }\end{array}$ \\
\hline Historia & E2 & $\begin{array}{l}\text { Profesor de historia, con } 40 \\
\text { años de edad y catorce años de } \\
\text { experiencia. }\end{array}$ \\
\hline Latín & E3 & $\begin{array}{l}\text { Profesor de latín, con } 56 \text { años } \\
\text { de edad y más de quince años } \\
\text { de experiencia. }\end{array}$ \\
\hline Química & E4 & $\begin{array}{l}\text { Profesor de química, con } 38 \\
\text { años de edad y diez años de } \\
\text { experiencia. }\end{array}$ \\
\hline Filosofía & E5 & $\begin{array}{l}\text { Profesor de filosofía, con } 43 \\
\text { años de edad y más de diez } \\
\text { años de experiencia. }\end{array}$ \\
\hline Lengua & E6 & $\begin{array}{l}\text { Profesora de lengua, con } 32 \\
\text { años de edad y siete años de } \\
\text { experiencia. }\end{array}$ \\
\hline Francés & E7 & $\begin{array}{l}\text { Profesora de Francés, con } 29 \\
\text { años de edad y menos de cinco } \\
\text { años de experiencia. }\end{array}$ \\
\hline Inglés & E8 & $\begin{array}{l}\text { Profesora de Inglés, con } 31 \\
\text { años de edad y siete años de } \\
\text { experiencia. }\end{array}$ \\
\hline Economía & E9 & $\begin{array}{l}\text { Profesor de economía, con } 58 \\
\text { años de edad y más de quince } \\
\text { años de experiencia. }\end{array}$ \\
\hline Física & E10 & $\begin{array}{l}\text { Profesora de física, con } 57 \text { años } \\
\text { de edad y más de quince años } \\
\text { de experiencia. }\end{array}$ \\
\hline
\end{tabular}

Fuente: elaboración propia

\subsection{Instrumentos}

Para la recogida de datos se utilizaron las siguientes técnicas:

3.2.1. Grupo de discusión: es una técnica que trata del estudio de saber lo que piensan y lo que comparten varios individuos con una reflexión conjunta en un espacio común (Mena y Méndez, 2009). En la sesión se cuidaron aspectos como luminosidad, espacio y temperatura. Se hizo una introducción y se eligió un moderador. Las cuestiones fueron: definición de con- flicto, existencia de conflictos entre el profesorado y la mejor manera de salir restablecido de estos. Todas estas aportaciones fueron recogidas en el cuestionario que se elaboró ad hoc.

3.2.2. Encuesta: cuestionario de elaboración propia compuesto por 35 ítems divididos en cuatro subescalas: (Anexo I).

1) Percepción del conflicto: conceptualización y forma de afrontamiento, con 10 ítems tales como inevitabilidad del conflicto, oportunidad de cambio, competición, huida y enriquecimiento personal.

2) Tipos de conflicto: con 10 ítems referidos a los tipos de conflictos más frecuentes (distribución de horarios, elección de asignaturas, franjas horarias, discrepancias con el equipo directivo y con la administración, compartir la misma asignatura, intereses y objetivos distintos, aprovechamiento de recursos materiales, valores distintos y falta de vocación docente).

3) Causas de los conflictos: con 8 ítems referidos a ideologías diferentes, promoción profesional, acceso a los recursos materiales, compartir la misma asignatura, diferencia de funciones, intereses distintos, comunicación deficiente e insatisfacción escolar.

4) Mecanismos de resolución: con 7 ítems tales como, buscar la ayuda de otros, informar al equipo directivo para que intervenga, asesoramiento de los sindicatos de enseñanza, intentar ocultarlo y que el tiempo lo resuelva, lo que se entiende por mediación como mecanismo útil y la posibilidad de crear un servicio de mediación para solucionar los conflictos.

Para ello se utilizó una escala tipo Likert de 4 puntos ( $1=$ nada de acuerdo a 4= muy de acuerdo), que consistió en conocer la reacción de los encuestados, al elegir uno de los cuatro puntos de la escala en forma de afirmaciones o juicios, para saber en qué medida estaban de acuerdo o desacuerdo con una afirmación y/o negación o pregunta en particular.

3.2.3. Entrevistas semiestructuradas: técnica complementaria al cuestionario. Se realizaron 10 entrevistas con 18 preguntas, cada vez más específicas a medida que avanzaba la entrevista a algunos miembros de los departamentos didácticos que no habían participado en las técnicas anteriores. Las preguntas se englobaron en los siguientes bloques: a) Percepción del conflicto, con 6 preguntas referidas al conflicto y sus consecuencias b) Tipos de conflictos más habituales entre el profesorado y equipo directivo, con 5 preguntas sobre infundios, falta de respeto, acusaciones inciertas, papel 
del equipo directivo ante los conflictos como agravante, mediador o negociador, las funciones de liderazgo y la falta de comunicación c) Causas de los conflictos, con 4 preguntas encaminadas a conocer las causas de los mismos, las consecuencias negativas como bajas por enfermedad y el apoyo entre el profesorado d) Mecanismos utilizados para su resolución, con 3 preguntas destinadas a conocerlos, las ventajas de la mediación, la formación sobre ésta y la posibilidad de implantar un servicio en el centro o fuera de él para la resolución de conflictos. Se utilizó grabadora con permiso de los entrevistados para su posterior transcripción.

\subsection{Procedimiento}

Se llevó a cabo una entrevista con el director para explicar la finalidad del estudio, describir los instrumentos y promover de forma voluntaria la colaboración del profesorado; se recogió la información mediante las técnicas descritas con anterioridad, ajustándonos al horario elegido por cada participante; se interpretaron los resultados cualitativos, se informatizaron los resultados cuantitativos y se realizaron los análisis de datos a través del programa SPSS versión 21 para Windows.

\subsection{Cuestiones éticas de los participantes}

Todas las técnicas empleadas en el estudio, estuvieron contempladas bajo las normas éticas que se incorporan al conocimiento científico, para garantizar una investigación éticamente responsable. Tal y como destaca Giménez (2014), se llevaron a cabo en la formulación de las preguntas del estudio: protección, selección de participantes y consentimiento informado. En el diseño y metodología en cuanto a conflicto de intereses, confidencialidad, privacidad y relaciones duales. $Y$ en los resultados respecto a informes y difusión. Con la garantía de preservar el anonimato de los participantes.

\section{RESULTADOS}

Los resultados se concretan a través de las técnicas empleadas. Por un lado, el análisis cuantitativo nos ha permitido obtener los datos requeridos en relación a los objetivos a través de la encuesta, utilizando una escala con fiabilidad media alta (Alfa de Cronbach de 0,74). Refiriéndonos a la validez de los ítems que componen la escala, destacamos que todos ellos cumplen el criterio de validez, ya que todos los elementos correlacionan con el total, de forma que si elimináramos alguno de ellos la fiabilidad total de la escala descendería.
Los resultados obtenidos del análisis cuantitativo han sido los siguientes:

a) Percepción del conflicto en el profesorado, mostrado en la Tabla IV.

\begin{tabular}{|c|c|}
\hline $\begin{array}{c}\text { TABLA IV. MEDIAS OBTENIDAS SOBRE LA } \\
\text { PERCEPCIÓN DEL CONFLICTO EN EL PROFESORADO }\end{array}$ & \\
\hline PERCEPCIÓN DEL CONFLICTO & MEDIA \\
\hline $\begin{array}{l}\text { En la relación entre profesores, es inevitable tener } \\
\text { conflictos }\end{array}$ & 2,58 \\
\hline En un conflicto una parte gana y otra pierde & 2,06 \\
\hline $\begin{array}{l}\text { Para no tener conflictos con nadie, lo mejor es } \\
\text { ceder }\end{array}$ & 1,61 \\
\hline $\begin{array}{l}\text { En un conflicto, es preciso luchar, para salir vence- } \\
\text { dor }\end{array}$ & 2,12 \\
\hline $\begin{array}{l}\text { La mejor manera de ayudar a un compañero que } \\
\text { tiene un conflicto, es quitarle } \\
\text { importancia }\end{array}$ & 1,91 \\
\hline $\begin{array}{l}\text { Los conflictos generan un desgaste mental y emo- } \\
\text { cional }\end{array}$ & 3,48 \\
\hline $\begin{array}{l}\text { A los conflictos se responde elevando el tono de } \\
\text { voz }\end{array}$ & 1,58 \\
\hline El conflicto nos lleva a un enriquecimiento personal & 1,97 \\
\hline
\end{tabular}

1) Desgaste emocional. La existencia de conflictos produce un mayor desgaste emocional en el profesorado $(x=3,5)$ que percibe como inevitables $(\dot{x}=2,6)$. Si comparamos las medias a nivel descriptivo, las puntuaciones obtenidas por la percepción del conflicto, destacamos que las medias más altas se sitúan en aquellos encuestados, que manifiestan estar muy de acuerdo con la existencia de conflictos y entienden que estos generan un desgaste mental y emocional. Los conflictos pueden ser una oportunidad para el cambio $(\dot{x}=2,4)$. Y como posibilidad de crecimiento personal ( $x=2)$.

El grupo de discusión, añadió que: "muchos de los conflictos escolares entre el profesorado podrían tener una pronta solución favorable a las partes". Afirmando que: "si no se encuentra el acuerdo, los conflictos quedan enquistados y se trasladan al plano personal, en perjuicio de la convivencia, afectando a toda la comunidad educativa".

Comentario expresado en las entrevistas semiestructuradas fue: "es un motor de cambio, lo importante es la gestión para obtener resultados positivos aunque provoca desgaste mental y emocional" (E1).

2) En un conflicto, es preciso luchar, para salir vencedor $\left({ }^{\prime} x=2,1\right)$. Esta forma de resolución queda recogida en los siguientes comentarios en cuanto a la formación así, uno de 
los casos manifiesta que: "cuando ha ocurrido un conflicto entre profesores, se ha llevado el caso al equipo directivo para buscar solución, aunque este en lugar de solucionar ha magnificado el conflicto porque se ha posicionado a favor de una parte, por carecer de formación" (E2). Otro opina que: "la formación en mediación debería ser impartida por expertos y no pedagogos de libro; no solo vendría bien la formación en mediación al equipo directivo sino también al resto del profesorado" (E5). En relación a las vías o los mecanismos de resolución de conflictos, uno de casos opina que: "la única vía para resolver conflictos es el equipo directivo, que no sabe hacerlo, la administración que hace que el conflicto se dilate en el tiempo y nunca llega la solución y los sindicatos de enseñanza que te escuchan pero que tampoco llegan a solucionar nada" (E4).

3) Hay diferencias de percepción del conflicto en relación con la variable sexo. Las mujeres $(x=1,46)$ consideran más que los hombres $(x=1,05)$ que los conflictos se solucionan ignorando a la otra parte. Esta diferencia es estadísticamente significativa $S=0.04<0,05$ (prueba T).

Igualmente la forma de evitar los conflictos por medio de ceder, es mayor en el caso de las mujeres $(x=2)$ que en los hombres $(x=1,36)$. Esta diferencia es estadísticamente significativa $\mathrm{S}=0.03<0,05$ (prueba $\mathrm{T}$ ).

4) El profesorado mantiene diferencias en relación a la edad: el grupo de más de 46 años obtiene medias más bajas que el resto en cuanto a la inevitabilidad de los conflictos (' $x=2,24)$ y en cuanto a considerar los conflictos como una oportunidad (' $x=2,19)$. Las diferencias con el resto de grupos son significativas.

La edad parece ser un factor que relativiza la existencia de conflictos en el profesorado. Este dato indica que aquel con más de 15 años de antigüedad, considera el conflicto como oportunidad para cambiar que aquel con menos experiencia.

b) Mecanismos de resolución de conflictos, se detalla en la Tabla $\mathrm{V}$.

Medias obtenidas sobre los mecanismos de resolución:

1) Ayuda de otros profesores para que intermedien en el asunto $(x=2,5)$. Encontramos en las respuestas, buscar la ayuda de otros profesores para que puedan mediar.

2) Solicitar apoyo en los sindicatos de enseñanza ( $x=2,3)$. Otra forma de resolver los conflictos, es pedir asesoramiento a los sindicatos de enseñanza.

\section{Tabla V. Mecanismos de Resolución de Conflictos}

\begin{tabular}{|c|c|}
\hline MECANISMOS DE RESOLUCION & MEDIA \\
\hline $\begin{array}{l}\text { Buscando la ayuda de otros, para que puedan } \\
\text { mediar }\end{array}$ & 2,36 \\
\hline Se informa al Equipo directivo para que intervenga & 2,18 \\
\hline $\begin{array}{l}\text { Se pide asesoramiento a los sindicatos de } \\
\text { enseñanza }\end{array}$ & 2,24 \\
\hline Se intenta que no se sepa. El tiempo lo resolverá & 1,70 \\
\hline $\begin{array}{l}\text { He oído hablar de mediación y considero que es un } \\
\text { mecanismo muy útil }\end{array}$ & 2,73 \\
\hline $\begin{array}{l}\text { La mediación favorece la comunicación entre las } \\
\text { partes }\end{array}$ & 3,24 \\
\hline $\begin{array}{l}\text { La creación de un servicio de mediación en el } \\
\text { Instituto, solucionaría muchos de } \\
\text { los conflictos entre el profesorado }\end{array}$ & 2,94 \\
\hline
\end{tabular}

3) Informar al equipo directivo $(x=2,2)$. Se solicita y se informa del conflicto al equipo directivo para que intervenga, mediando entre las partes.

4) Permitir que el tiempo resuelva la situación $(x=1,7)$. El tiempo es otra forma de resolución, se intenta ocultar el conflicto y que se resuelva con el paso del tiempo.

5) Hay diferencias significativas respecto al sexo, las mujeres prefieren de forma significativa intentar que el conflicto no se sepa y que lo resuelva el paso del tiempo. La ' $x$ de mujeres en el ítem $=2.00$ y la de los hombres $=1.45$. Esta diferencia es significativa estadísticamente $(S=0.039<0.05)$. Las diferencias son también significativas en relación al ítem de resolución del conflicto, informando al equipo directivo en función de la edad, de forma que el profesorado de más edad prefiere no resolver el conflicto acudiendo al equipo directivo.

6) La forma de resolver los conflictos tiene diferencias en cuanto a informar al equipo directivo, en relación a la edad. La ' $x$ del grupo de edad 36-45 años es $=2.67$ y la de profesores del grupo de edad entre 46-60 años es de 1.86. ( $S=0.039<$ 0.05). El profesorado de más edad prefiere no informar a la dirección. Sin embargo, no hemos encontrado diferencias en las formas de solucionar los conflictos en el centro respecto a la experiencia como docente. No hay diferencias significativas de las medias: ni entre los grupos de 0-5 años, de 6 a 15 ni de más de 15 años de experiencia. Estos datos parecen indicar que, la edad es un factor que influye en la forma de resolver el conflicto de forma que, el profesorado con menos edad acude con mayor incidencia que los mayores a la vía de la autoridad. Sin embargo, la experiencia como profesor no influye en la forma en que entienden que los conflictos deben resolverse. 
c) Los tipos de conflictos, se muestra en la Tabla VI.

\begin{tabular}{|lc|}
\hline \multicolumn{2}{|c|}{ TABLA VI. MEDIAS OBTENIDAS SOBRE LOS TIPOS } \\
DE CONFLCTOS ENTRE EL PROFESORADO
\end{tabular}

1) Los profesores consideran las discrepancias con el equipo directivo (' $x=3,4)$ y con la administración (' $x=3,3$ ) como los conflictos más relevantes entre profesorado. Presentamos un segmento de un caso. "Se producen conflictos con el equipo directivo; este no potencia con igual celo todas la enseñanzas del centro (o así se percibe), la información no fluye como debería, se interpreta de forma distinta la aplicación de las normas o la legislación; se tiene la impresión de que el equipo directivo es una parte más de la administración y no defiende al claustro de la manera más adecuada" (E9). Otro añadió que: "en muchos casos, el equipo directivo es el causante de los conflictos y nunca negocia, impone; en caso de conflictos se inhibe" (E3). Coincidiendo con la misma pregunta, otro caso aseguraba que: "el equipo directivo es el causante de los conflictos por incompetencia, siendo alguno de estos sencillos de resolver; algunos han repercutido incluso en que algún compañero haya sido desplazado" (E2). Otro entrevistado opinó que: "el equipo directivo solo supervisa y aunque intenta mediar, no sabe y a menudo escucha pero no le interesa oír lo que se le dice" (E5). La mayoría de los entrevistados tiene una visión negativa del equipo directivo por falta de comunica- ción y función de liderazgo; siendo uno de los principales conflictos mencionados por los participantes.

2) La adjudicación de los horarios de clase y las franjas horarias $(x=2,9)$. Este resultado se ve reflejado en el grupo discusión cuando, por ejemplo, se señala que: "En muchos casos, el equipo directivo es el causante de estos conflictos y nunca negocia, impone; en caso de conflictos se inhibe". Coincidiendo con la misma pregunta, otro entrevistado añadió que: "el equipo directivo ha sido el causante de los conflictos por incompetencia, siendo alguno de estos sencillos de resolver y han repercutido incluso en que algún compañero haya sido desplazado" (E2). Otro caso opina que: "el equipo directivo solo supervisa y aunque intenta mediar, no sabe y a menudo escucha pero no le interesa oír lo que se le dice" (E5). La mayoría de los entrevistados tiene una visión negativa del equipo directivo.

3) Problemas de comunicación ( $x=2,8)$ En las entrevistas/ grupo discusión se señala, por ejemplo, que: "una de las causas que genera conflictos es la falta de comunicación y la unión entre este colectivo, el problema se produce cuando hay relación profesional y se persiguen los mismos objetivos e intereses" (E6). Otro participante señaló que: "se han dejado correr informaciones falsas, o lo que es lo mismo, no se ha aclarado o informado cuando era necesario" (E3).

Otro caso opinó que: "apenas hay convivencia entre el profesorado debido a los recortes en educación, el profesorado dispone de pocas horas de trabajo personal o tiempo libre, esto da lugar a desconocimiento sobre un determinado conflicto, por lo que la escasez de información en lugares inapropiados (corrillos) hace que se manipule o contamine, generando malentendidos que provocan magnitud del conflicto, lejos de la solución" (E7).

4) Uso de recursos materiales ( $x=2,4)$, a problemas de incompatibilidad de intereses $(\dot{x}=2,4)$ y a valores contrapuestos $(\dot{x}=2,3)$. En las entrevistas/grupo de discusión, por ejemplo uno de los participantes señaló que: "el conflicto se produce por intereses opuestos; siendo la propia legislación educativa la que permite que dos departamentos compitan por un mismo tipo de alumnado" (E8). Otro caso añadió que: "el colectivo de profesores no es capaz de consensuar acciones de respuesta unificada" (E1). Otro afirmó que: "cada uno va a lo suyo" (E10).Sin embargo, cuando se preguntó sobre las bajas por enfermedad, debidas a conflictos, refieren no tener constancia de ningún caso. Aunque un caso contestó que: "el estrés aumenta con los conflictos, quizá porque no hay 
manera de encauzar una respuesta común e incluso algunos han provocado enfermedades y bajas laborales" (E9).

5) La promoción personal $(\dot{x}=2,2)$. Parece que el profesorado está menos preocupado por la posibilidad de competir por ascensos dentro del centro educativo.

Así, uno de los casos respondió que: "apenas hay convivencia entre el profesorado debido a los recortes en educación; se dispone de pocas horas de trabajo personal o tiempo libre, poco importa la promoción dentro del centro. La falta de tiempo para la convivencia, no ofrece información sobre un determinado conflicto o asunto, por lo que la escasez de información en lugares inapropiados (corrillos) hace que se manipule o contamine, dando lugar a malentendidos que provocan la magnitud del conflicto, lejos de la solución" (E10).

6) Los conflictos menos presentes en el profesorado. Referidos a temas ideológicos $(\dot{x}=2,1)$ de vocación $(\dot{x}=2)$ o de reparto de funciones $(\dot{x}=2)$ y asignaturas $(\dot{x}=1,9)$. Uno de los participantes añadió que: "cuando ocurre un conflicto entre compañeros, es preciso que éste no adquiera un carácter personal o ideológico sino que sea de tipo exclusivamente escolar o profesional como reparto de funciones o falta de vocación, siendo este tipo de conflictos escasos" (E6).

Por último, comprobamos que no existían diferencias significativas sobre los tipos y las causas de los conflictos en función de las variables sexo, edad, experiencia docente o titulación.

\section{CONCLUSIONES Y DISCUSIÓN}

A modo de conclusión podemos decir que, el conflicto en las relaciones humanas es inevitable (Martínez-Otero, 2001), lo que genera un desgaste emocional en el profesorado. No obstante, es considerado como oportunidad de cambio, coincidiendo con Pérez y Pérez de Guzmán (2011), que puede llevar al crecimiento personal al tener que afrontar y superar situaciones conflictivas con repercusión en la autoestima, entendimiento y convivencia.

Los tipos de conflictos más frecuentes son los producidos con la dirección del centro y con la administración educativa en consonancia con Muñoz y Méndez (2017), por una comunicación deficiente. Otros conflictos son las preferencias en las franjas de los horarios, el uso de recursos materiales, incompatibilidad de intereses y valores contrapuestos. Coincidiendo con los datos extraídos de la memoria del sindicato "Asociación Nacional de Profesio- nales de la Enseñanza" (2011-12). La promoción personal también genera conflicto mientras que, los problemas relativos a ideologías o reparto de asignaturas no tienen gran impacto. La forma en que se tiende a responder al conflicto es la competitividad, de forma que, el que pierde intentará producir una nueva situación para restablecer su posición. La percepción en la resolución de conflictos más común, es recurrir a la ayuda de otros para que intermedien, solicitar la ayuda de organizaciones sindicales, o bien, recurrir al equipo directivo. Con diferencias en cuanto al sexo ya que, las mujeres tienden, en mayor medida que los hombres, a resolver el conflicto por medio de ignorar a la otra parte, así como, a utilizar la estrategia de ceder para evitar la situación conflictiva.

Igualmente, se han encontrado diferencias en cuanto a la variable edad. El profesorado con más edad considera que los conflictos pueden evitarse y ser considerados en su dimensión de oportunidad, en mayor medida que los más jóvenes. Sin embargo, no se aprecian diferencias en las formas de solucionar los conflictos en el centro respecto a la experiencia docente.

La falta de comunicación o cierto hermetismo del equipo directivo, puede generar comunicación desigual en lugares inapropiados, que en ocasiones, produce malentendidos, percepciones erróneas y luchas de poder, supuestos sin comprobación, estereotipos, expectativas no realistas, rumores y recuerdos de conflictos sin resolver, de diferente intensidad e índole; lo que produce bajas escolares con repercusión en la organización del IES, en consonancia con Prieto y Bermejo (2006) produciendo además, malestar docente (Sánchez, March y Ballester, 2015;González, 2017).

Este estudio de caso, representa una muestra de las múltiples situaciones conflictivas que se pueden presentar en los centros educativos, bajo una aparente imagen de falta de conflictividad. Aunque, coincidiendo con Tahull y Montero (2015), los estudios sobre este tema son escasos, lo que nos ha supuesto una limitación, pero también un estímulo para seguir investigando con una muestra más amplia.

\section{REFERENCIAS BIBLIOGRÁFICAS}

Asociación Nacional de Profesionales de la Enseñanza (2012). Memoria Estatal del Defensor del Profesor. Curso $2011-$ 2012. Disponible en: https://eldefensordelprofesor.es/openFile.php?link ...defensor-del-profesorado

Arias, W. L. y Jiménez, N. A. (2013). Síndrome de burnout en docentes de Educación Básica Regular de Arequipa. Revista Educación, 22(42), 53-76. 
Benito, B. (2006). Las relaciones interpersonales de los profesores en los centros educativos, Libro de actas de la XI Conferencia de Sociología de la Educación (pp. 218-219). Salamanca: Universidad de Salamanca.

Camacho, A. y Mayorga, D. R. (2017). Riesgos laborales psicosociales. Perspectiva organizacional, jurídica y social. Revista Prolegómenos Derechos y Valores, 20, (40), 159172.

Galdeano, H., Godoy, P., y Cruz, I. (2007). Factores de riesgo psicosocial en profesores de educación secundaria. Arch Prev Riesgos Labor, 10(4), 174-180.

Giménez, V. M. (2014). Cuestiones éticas en la investigación en Trabajo Social y estrategias para una investigación éticamente responsable. AZARBE, Revista Internacional De Trabajo Social Y Bienestar, (3). Disponible en: https://revistas.um.es/azarbe/article/view/19854.

González, N. (2017). Estudio de las posibles causas del malestar de los profesores de secundaria desde su formación inicial. Tesis doctoral. Universidad Complutense de Madrid.

Hermosa, A. (2006). Satisfacción laboral y síndrome de" burnout" en profesores de educación primaria y secundaria. Revista Colombiana de Psicología, 15(1), 81-89.

Jares, X. (2001). Educación y Conflicto. Madrid: Popular.

Martínez-Otero, V. (2001). Convivencia escolar: problemas y soluciones. Revista complutense de Educación, 12 (1), 295-318.

Mena, A.M. y Méndez, J.M. (2009). La Técnica de grupo de discusión en la investigación cualitativa. Aportaciones para el análisis de los procesos de interacción. Revista Iberoamericana de Educación, 49(3), 1-2.
Merlano, S. (2011). Conflictos en el campo laboral. Estrategias para manejarlo. Contribuciones a las Ciencias Sociales. Disponible en: www.eumed.net/rev/cccss/11/

Muñoz-Méndez, T., Gómez-Mármol, A. y Sánchez-Alcaraz, J. S. (2017). Satisfacción laboral en los docentes de educación infantil, primaria y secundaria. Gestión de la Educación, 7(1), 161-177.

Pérez, G. y Pérez de Guzmán, M.V. (2011). Aprender a convivir: el conflicto como oportunidad de crecimiento (189). Madrid: Narcea.

Prieto, M. y Bermejo, L. (2006). Contexto laboral y malestar docente en una muestra de profesores de Secundaria. Revista de Psicología del Trabajo y de las Organizaciones, 22(1), 45-73.

Redorta, J. (2006). Emoción y conflicto. Aprenda a manejar las emociones. Barcelona: Paidós.

Sánchez, D., March, M., y Ballester, L. (2015). Malestar social y malestar docente: una investigación sobre el síndrome de desgaste profesional burnout y su incidencia socioeducativa. Revista Aula, 21, 245-257. DOI: http://dx.doi. org/10.14201/aula201521245257

Tahull, J. y Montero, Y. (2015). Malestar en la escuela. Conflictos entre profesores. Revista Educar, 51(1), 168-188.

Tamayo, M. (2002). El proceso de la investigación científica. México D.F: Limusa

Torres, J. A. (2013). Análisis del grado de satisfacción del profesorado de educación secundaria en el desarrollo de su labor docente. Contextos educativos. Revista de educación, (13), 27-42.

Vinyamata, E. (2003). Aprender del conflicto: Conflictología y educación. Barcelona: Graó. 


\section{ANEXOS}

\section{Anexo I}

\section{CUESTIONARIO PARTICIPANTES}

Gracias por el tiempo empleado para completar esta encuesta. No escriba su nombre ni lo firme. Es ANONIMO. Las preguntas están destinadas a conocer las causas y los tipos de conflictos entre el profesorado. Para llevar a cabo una Investigación para la Universidad de Murcia.

\section{DATOS PERSONALES Y PROFESIONALES:}

\begin{tabular}{|c|c|}
\hline $\begin{array}{l}\text { 1.- SEXO: } \\
\square \text { Hombre } \quad \square \text { Mujer }\end{array}$ & 2.- EDAD: __ años \\
\hline $\begin{array}{l}\text { 3.- DOCENCIA } \\
\text { (*Nota: puede señalar más de uno): } \\
\square \text { C. Formativo } \quad \square \text { ESO } \square \text { Bachillerato }\end{array}$ & $\begin{array}{l}\text { 4.- Tipo de centro: } \\
\square \text { Público } \quad \square \text { Privado } \quad \square \text { Concertado }\end{array}$ \\
\hline $\begin{array}{l}\text { 5.- SITUACIÓN ACTUAL COMO PROFESOR: } \\
\square \text { Destino definitivo. No Años: } \\
\square \text { Sustitución. No Años: } \\
\square \text { Otras. No Años: }\end{array}$ & $\begin{array}{l}\text { 6.- TITULACIÓN ACADÉMICA } \\
\text { (*Nota: puede señalar más de uno): } \\
\square \text { Licenciado } \\
\square \text { Profesor Técnico de F.P. } \\
\square \text { Otras }\end{array}$ \\
\hline $\begin{array}{l}\text { 7.- EXPERIENCIA DOCENTE: } \\
\square \text { Menos de cinco años } \quad \square \text { Entre seis y quince años } \\
\square \text { Más de quince años }\end{array}$ & $\begin{array}{l}\text { 8.- SATISFACCIÓN CON SU LABOR DOCENTE: } \\
\square 1 \quad \square 2 \quad \square 3 \quad \square 4 \quad \square 5 \\
1 \text { (Ninguna) } 2 \text { (Poca) } 3 \text { (Regular) } 4 \text { (Bastante) } 5 \text { (Mucha) }\end{array}$ \\
\hline
\end{tabular}

Por favor, lea atentamente la frase de la izquierda y conteste en la columna de la derecha, marcando una cruz en la casilla correspondiente, según esta opciones: 1 (nada de acuerdo) 2 (algo de acuerdo) 3 (de acuerdo) 4 (muy de acuerdo)

\section{PREGUNTAS}

\begin{tabular}{|c|c|c|c|c|}
\hline \multirow{2}{*}{$\begin{array}{l}\text { PERCEPCIÓN DEL CONFLICTO } \\
\text { Sobre los conflictos opino que... }\end{array}$} & \multicolumn{4}{|c|}{ OPCIONES } \\
\hline & 1 & 2 & 3 & 4 \\
\hline 1.-En la relación entre profesores, es inevitable tener conflictos & $\square$ & $\square$ & $\square$ & $\square$ \\
\hline 2.-Los conflictos son oportunidades de cambio & $\square$ & $\square$ & $\square$ & $\square$ \\
\hline 3.-En un conflicto una parte gana y otra pierde & $\square$ & $\square$ & $\square$ & $\square$ \\
\hline 4.- Los conflictos se solucionan ignorando a la otra parte & $\square$ & $\square$ & $\square$ & $\square$ \\
\hline 5.- Para no tener conflictos con nadie, lo mejor es ceder & $\square$ & $\square$ & $\square$ & $\square$ \\
\hline 6.- En un conflicto, es preciso luchar, para salir vencedor & $\square$ & $\square$ & $\square$ & $\square$ \\
\hline 7.- La mejor manera de ayudar a un compañero que tiene un conflicto, es quitarle importancia & $\square$ & $\square$ & $\square$ & $\square$ \\
\hline 8.- Los conflictos generan un desgaste mental y emocional & $\square$ & $\square$ & $\square$ & $\square$ \\
\hline 9.- A los conflictos se responde elevando el tono de voz & $\square$ & $\square$ & $\square$ & ㅁ \\
\hline 10.- El conflicto nos lleva a un enriquecimiento personal & $\square$ & $\square$ & $\square$ & $\square$ \\
\hline \multirow{2}{*}{$\begin{array}{l}\text { TIPOS DE CONFLICTOS } \\
\text { Los conflictos más frecuentes entre el profesorado son... }\end{array}$} & \multicolumn{4}{|c|}{ OPCIONES } \\
\hline & 1 & 2 & 3 & 4 \\
\hline 11.- Distribución de horarios. & $\square$ & $\square$ & $\square$ & $\square$ \\
\hline 12.- Elección de asignaturas que compiten por un alumnado concreto. & $\square$ & $\square$ & $\square$ & $\square$ \\
\hline 13.- Franjas horarias en las que se desea impartir las asignaturas. & $\square$ & $\square$ & $\square$ & $\square$ \\
\hline 14.- Discrepancias con el equipo directivo. & $\square$ & $\square$ & $\square$ & $\square$ \\
\hline 15.- Discrepancias y desacuerdos con la Administración. & $\square$ & $\square$ & $\square$ & $\square$ \\
\hline 16.- Compartir la misma asignatura. & $\square$ & $\square$ & $\square$ & $\square$ \\
\hline 17.-Intereses y objetivos distintos. & $\square$ & $\square$ & $\square$ & $\square$ \\
\hline 18.- Aprovechamiento de los recursos materiales & $\square$ & $\square$ & $\square$ & $\square$ \\
\hline 19.- Valores distintos & $\square$ & $\square$ & $\square$ & $\square$ \\
\hline 20.- Falta de vocación docente & $\square$ & $\square$ & $\square$ & $\square$ \\
\hline
\end{tabular}




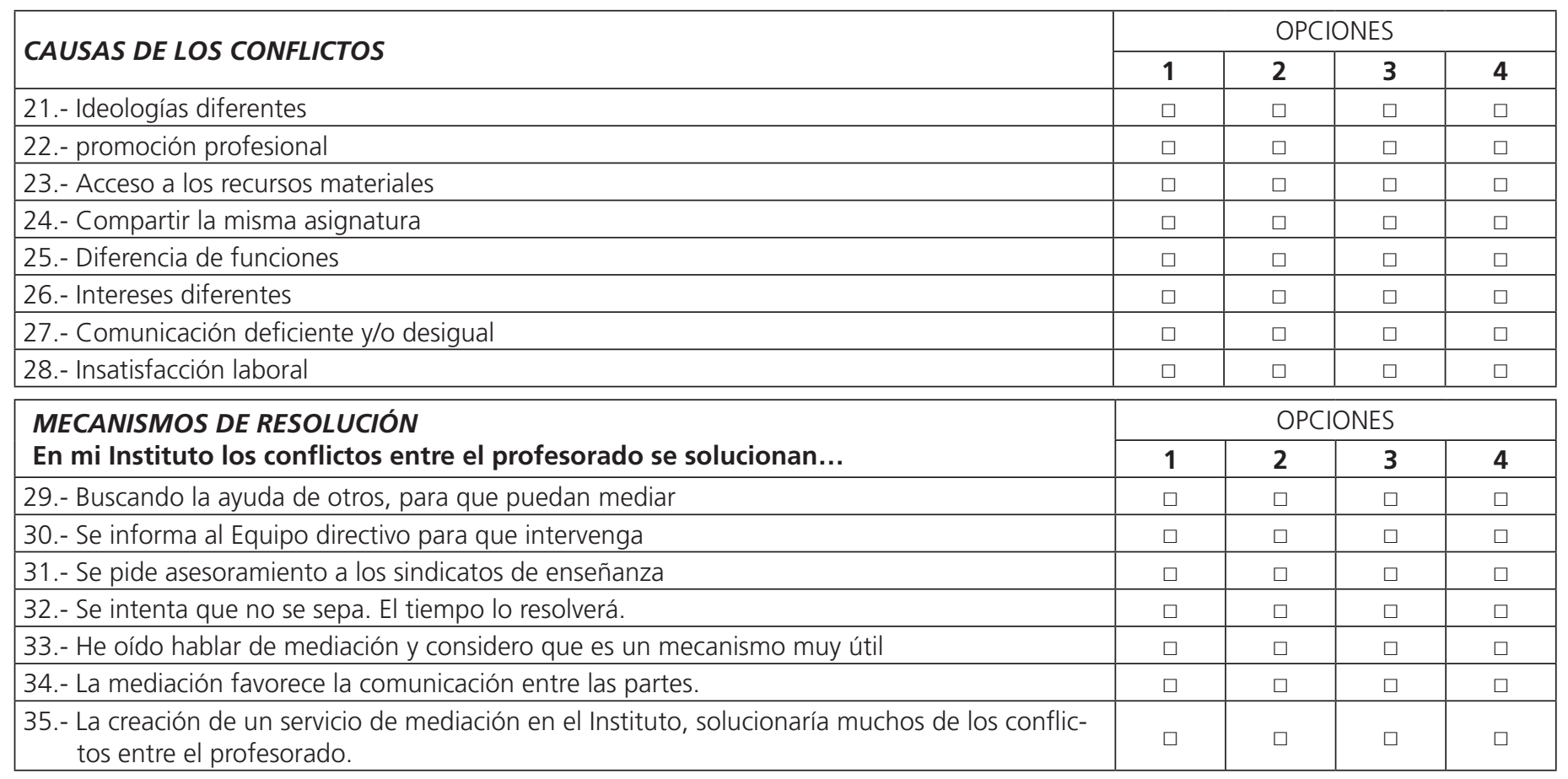

\section{Gracias por su colaboración}

